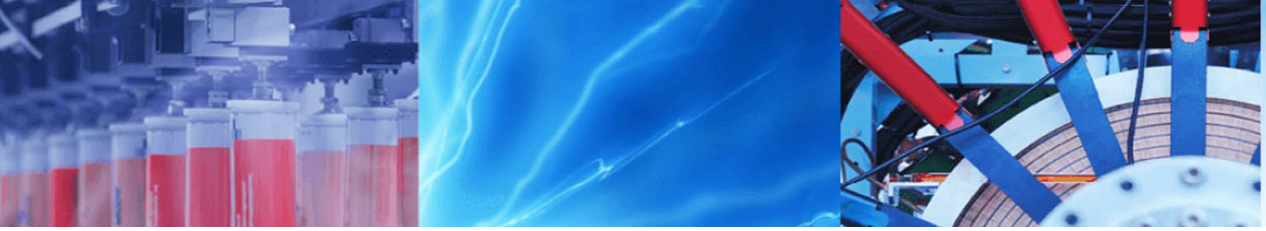

Research Article

\title{
Raman spectroscopy and transmission electron microscopy of long linear atomic carbon chains generated by field electron emission accompanied with electrical discharge of carbon nanotube films
}

\author{
Koji Asaka ${ }^{1}$ (D) Satoshi Toma ${ }^{1} \cdot$ Yahachi Saito $^{1,2}$
}

(c) Springer Nature Switzerland AG 2019

\begin{abstract}
Carbyne is a carbon allotrope whose structure is a one-dimensional chain of sp-hybridized carbon atoms. Carbyne's mechanical and electrical properties, as predicted by theoretical studies, have attracted great interest because they would lead to many promising applications. Thus, much effort has been devoted to the synthesis of carbyne. Long linear atomic carbon chains encapsulated in carbon nanotubes have recently been produced by high-temperature heat treatment of double-wall carbon nanotubes (DWCNTs). Here, we present an alternative approach to produce long linear carbon chains: field electron emission accompanied by electrical discharge from single-wall carbon nanotube (SWCNT) films. Raman spectroscopy and transmission electron microscopy were performed on SWCNT films after the electrical discharge during field electron emission. The results showed that a large number of long linear carbon chains were formed within the SWCNTs and DWCNTs. For DWCNTs with an inner diameter of $0.7 \mathrm{~nm}$, the atomic carbon chains lay directly along the central tube axis. However, for SWCNTs with an inner diameter of $1.0 \mathrm{~nm}$, the encapsulated carbon chains were bent in some places and positioned close to the nanotube wall, away from the central tube axis.
\end{abstract}

Keywords Carbon nanotube · Carbyne · Field electron emission · Transmission electron microscopy · Raman spectroscopy

\section{Introduction}

Carbyne is an allotrope of carbon that consists of an infinitely long chain of sp-hybridized carbon atoms. The structure of carbyne can take on two possible configurations: (1) cumulene, which has repeating double bonds throughout the chain, and (2) polyyne, which has alternating single and triple bonds. Cumulene may spontaneously transform into polyyne through a Peierls distortion [1, 2]. It has been theoretically predicted that carbyne possesses an exceedingly high stiffness and Young's modulus [3-5], a potentially high thermal conductivity [6-8], and unusual electrical transport properties [9-12]. Moreover, carbyne has attracted great interest because it can potentially be used in many different applications, such as switching nanodevices [13, 14], spintronic nanodevices [15-17], and hydrogen storage [18].

The existence of carbyne was debated for many decades until the successful synthesis of a linear acetylenic carbon chain with end-capping groups [19]. The organic synthesis technique yielded an acetylenic carbon chain consisting of 44 atoms [20]. In addition, transmission electron microscopy has also identified short free-standing carbon chains directly forming from carbon nanotubes [21-25] and graphene [26-36] under electron-beam irradiation and application of electrical current. Furthermore, the electric current passing through carbon chains $[25,30$,

\footnotetext{
$\triangle$ Koji Asaka, asaka@nuqe.nagoya-u.ac.jp|'Department of Applied Physics, Graduate School of Engineering, Nagoya University, Furo-cho, Chikusa-ku, Nagoya 464-8603, Japan. ${ }^{2}$ Present Address: Toyota Physical and Chemical Research Institute, 41-1, Yokomichi, Nagakute 480-1192, Japan.
}

SN Applied Sciences (2019) 1:493 | https://doi.org/10.1007/s42452-019-0481-9 
$32,35]$ and their tensile force at fracture [23] were measured in situ.

Isolated long linear atomic carbon chains have not yet been observed under ambient conditions because of their high chemical reactivity and extreme instability. However, it has been reported that there is the possibility to grow such chains in multiwall carbon nanotubes (MWCNTs) by the arc discharge method in hydrogen gas [37], liquid nitrogen [38, 39], argon gas [40], or helium gas [41, 42]. Carbon chains have also been fabricated by the high-temperature fusion reaction of molecules such as $\mathrm{C}_{10} \mathrm{H}_{2}$ [43] and adamantane [44] within double-wall carbon nanotubes (DWCNTs), whose linear cavity can provide a space for encapsulating and stabilizing long linear carbon chains. Furthermore, Shi et al. [45] have reported long linear carbon chains consisting of more than 6000 atoms, synthesized within DWCNTs by high-temperature heat treatment under a high vacuum.

Recently, we have found out the presence of long linear carbon chains after electrical discharge during field electron emission from single-wall carbon nanotube (SWCNT) films [46], which is different from the previous method by arc discharge and high-temperature heat treatment. The present reproducible synthesis method may provide a new approach to the bulk production of long linear carbon chains. In this paper, we report the field emission properties accompanied by the electrical discharge of SWCNT films that lead to the formation of long linear carbon chains encapsulated within carbon nanotubes, and characterize these carbon chains by Raman spectroscopy and electron microscopy.

\section{Experimental}

SWCNT films synthesized by an enhanced direct injection pyrolytic synthesis method (Meijo Nano Carbon Co., Ltd., film thickness; approximately $40 \mu \mathrm{m}$, a nominal diameter of SWCNT; 1-3 nm) were used in the present study. In the as-prepared SWCNT films, DWCNTs and triple-wall carbon nanotubes were contained as minor constituents. Field electron emission experiments were conducted on these SWCNT films at room temperature under a vacuum pressure of $8.3 \times 10^{-6} \mathrm{~Pa}$ in a parallel-plate configuration. The distance between the electrodes was $0.16 \mathrm{~cm}$. The field emission specimens were prepared by cutting the SWCNT films into roughly $5 \times 5 \mathrm{~mm}$ pieces, and attaching each piece onto the surface of an aluminum cathode using an Ag adhesive paste (Dotite D-500, Fujikura Kasei Co., Ltd.). The morphology and structure of the specimens were observed by scanning electron microscopy (SEM; FEI Quanta 200 FEG, $15 \mathrm{kV}$ ) and transmission electron microscopy (TEM; JEOL JEM-2010, 120 kV). The TEM specimens were prepared by dropping SWCNTs dispersed in isopropyl alcohol onto a microgrid. Raman characterization of the specimens was carried out with a Renishaw inVia Reflex Raman microscope using a laser excitation source with a wavelength of $532 \mathrm{~nm}$ at room temperature (measurement conditions; laser power $0.15 \mathrm{~mW}$, exposure time $10 \mathrm{~s})$.

\section{Results and discussion}

The field emission characteristics of the SWCNT films were measured at room temperature under a vacuum pressure of $8.3 \times 10^{-6} \mathrm{~Pa}$. Figure $1 \mathrm{a}$ plots current-voltage curves obtained during field emission from an SWCNT film. The first field emission experiment started with an emission current of more than $10 \mathrm{nA}$ at a voltage of $354 \mathrm{~V}$ and increased up to $962 \mu \mathrm{A}$ at $868 \mathrm{~V}$ (arrowhead a), at which point an electrical discharge occurred and the applied voltage was immediately made to decrease to zero. The
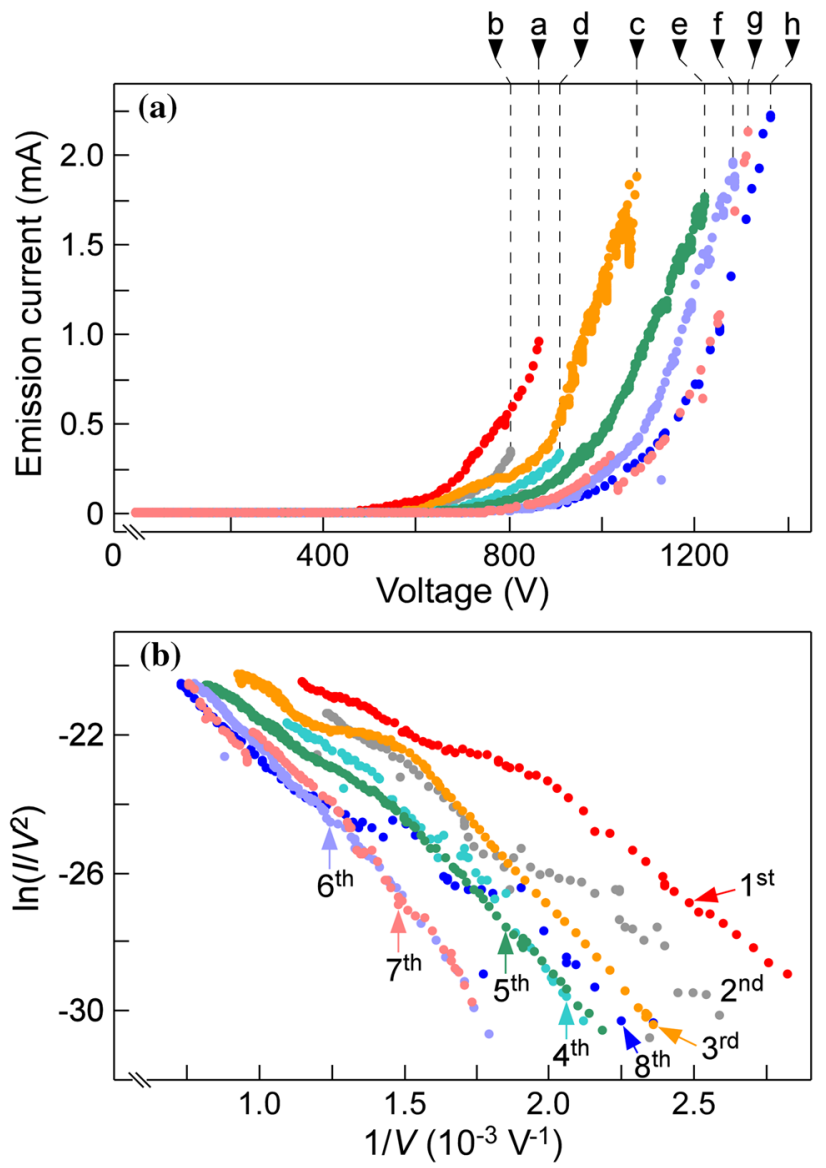

Fig. 1 a Current-voltage curves measured during field emission from an SWCNT film. Arrowheads a-h indicate the discharge voltages from the first to eighth field emission experiment, respectively. b Fowler-Nordheim plots obtained from (a)

\section{SN Applied Sciences}


process of field emission followed by electrical discharge was repeated eight times (see Fig. 1a). The voltages indicated by arrowheads a-h in Fig. 1a show, respectively, the discharge voltages from the first to eighth field emission experiment, which tend to increase with the number of repetitions. The turn-on voltage (defined here as the applied voltage to obtain an emission current of $10 \mathrm{nA}$ ) increased as the field emission experiment proceeded (see Table 1), indicating that it became increasingly more difficult to emit electrons during field emission under the same applied voltage, owing to morphological and destructive changes in the SWCNT film by the electrical discharge, as shown in Fig. 2b. According to the Fowler-Nordheim equation [47], the emission current $/$ is given by

$I=1.4 \times 10^{-6} \frac{A \beta^{2} V^{2}}{d^{2} \phi} \exp \left(-6.49 \times 10^{7} \frac{d \phi^{3 / 2}}{\beta V}+\frac{9.8}{\phi^{1 / 2}}\right)$

where $A$ is the emitting area, $\beta$ is the field enhancement factor, $V$ is the applied voltage, $d$ is the distance between electrodes, and $\phi$ is the work function of the emitter. The graph obtained by plotting $\ln \left(I / V^{2}\right)$ against $1 / V$ is called the Fowler-Nordheim plot and is used to analyze the emission current-voltage curve. Figure $1 \mathrm{~b}$ shows the Fowler-Nordheim plots obtained from the current-voltage curves in Fig. $1 \mathrm{a}$, assuming $\phi=4.6 \mathrm{eV}$ for graphite and using a $d$ value of $0.16 \mathrm{~cm}$. In all the Fowler-Nordheim plots in Fig. $1 \mathrm{~b}$, the slopes of the plots in the high-voltage regions deviate from those in the low-voltage regions, i.e., a reduction in emission current occurs at high voltages. The field enhancement factor for each field emission experiment is estimated from the slopes in the low-voltage regions in Fig. $1 \mathrm{~b}$ (see results in Table 1). The enhancement factors decreased with each repetition of the field emission experiment owing to the morphological changes that the SWCNT film underwent due to the electrical discharge during field electron emission.

Figure $2 \mathrm{a}, \mathrm{b}$ shows SEM images of an as-prepared SWCNT film and an SWCNT film after electrical discharge repeated eight times in the field emission experiment as shown in Fig. 1, respectively. The surface morphology of the as-prepared SWCNT film, which is composed of entangled SWCNTs, is homogeneous as shown in Fig. 2a, while after the electrical discharge, parts of the SWCNT film are
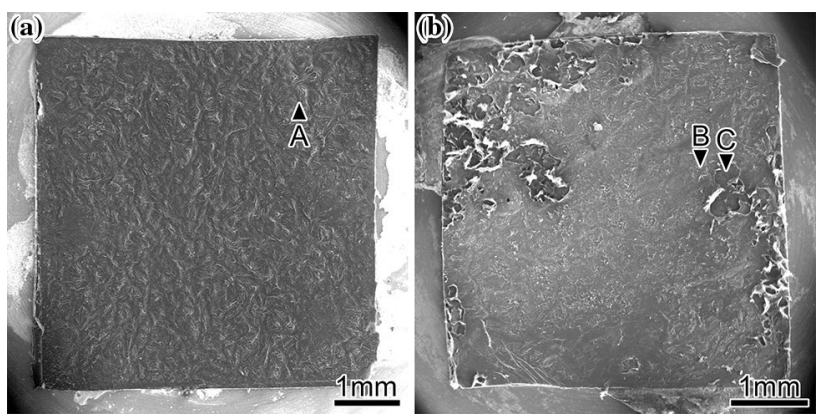

Fig. 2 SEM images of $\mathbf{a}$ an as-prepared SWCNT film and $\mathbf{b}$ an SWCNT film after electrical discharge in the field emission experiment

damaged and torn up, resulting in the formation of holes, as seen in Fig. 2b. These significant morphological changes suggest that the electrical discharge during field emission causes structural transformation of the SWCNTs.

In order to determine the influence of the damage in the SWCNT films, they were examined by Raman spectroscopy. Figure 3a shows a Raman spectrum of the asprepared SWCNT film, measured from the region indicated by arrowhead A in Fig. 2a. Two large peaks appear at 1593 and $2677 \mathrm{~cm}^{-1}$, which are assigned to the $G$ and $2 \mathrm{D}$ bands for SWCNTs, respectively. The peaks in the range lower than $\sim 400 \mathrm{~cm}^{-1}$ are the radial breathing modes (RBMs) for SWCNTs. At above $200 \mathrm{~cm}^{-1}$, the spectral noise is large as shown in Fig. 3 and the RBMs were hard to be assigned accurately. The D band for SWCNTs is observed at $1342 \mathrm{~cm}^{-1}$, and its peak intensity, $I_{D}$, is much smaller than that of the $G$ band, $I_{G}$. The degree of structural disorder for carbon networks in SWCNTs, $P$, is estimated by $P=I_{\mathrm{D}} /\left(I_{\mathrm{D}}+I_{\mathrm{G}}\right) \times 100 \%$. The $P$ value for the as-prepared SWCNT film in Fig. $4 a$ is $1.5 \%$. Figure $3 b$, c shows Raman spectra from the non-damaged and damaged regions of an SWCNT film after electrical discharge, indicated by arrowheads $B$ and $C$ in Fig. 2b, respectively. The $P$ values for the SWCNT films after electrical discharge are 4.5 (Fig. 3b) and $9.0 \%$ (Fig. 3c), suggesting that structural disorder is introduced into the carbon networks in the SWCNTs upon electrical discharge. In the Raman spectrum from the damaged region, a sharp peak and a broad peak appear at $1863 \mathrm{~cm}^{-1}$ and $\sim 600 \mathrm{~cm}^{-1}$, respectively, as seen

Table 1 Discharge voltage, turn-on voltage, and field enhancement factor obtained from Fig. 1

\begin{tabular}{lllllllll}
\hline & 1st & 2nd & 3rd & 4th & 5th & 6th & 7th & 8th \\
\hline Discharge voltage (V) & 867.7 & 807.3 & 1075.7 & 910.6 & 1223.4 & 1286.7 & 1316.5 & 1366.2 \\
Turn-on voltage (V) & 354.2 & 386.0 & 423.6 & 470.8 & 457.3 & 557.8 & 575.2 & 423.4 \\
$\begin{array}{l}\text { Field enhancement } \\
\text { factor } \times 10^{4}\end{array}$ & $1.501 \pm 0.003$ & $1.234 \pm 0.031$ & $1.098 \pm 0.001$ & $0.903 \pm 0.008$ & $1.168 \pm 0.001$ & $0.916 \pm 0.005$ & $0.885 \pm 0.008$ & $1.179 \pm 0.033$ \\
\hline
\end{tabular}




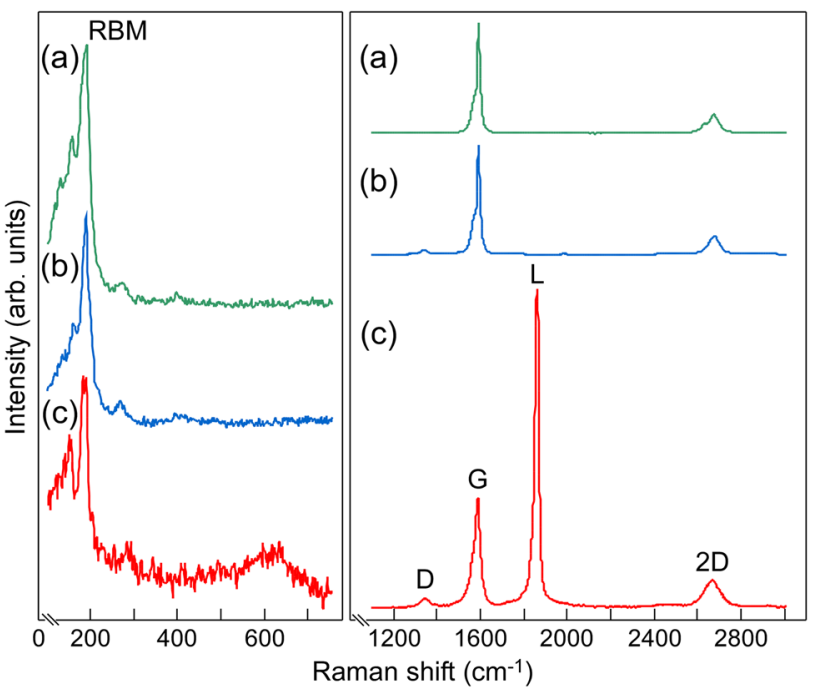

Fig. 3 Raman spectra measured from a the region indicated by arrowhead A in Fig. 2a for the as-prepared SWCNT film, b and $\mathbf{c}$ non-damaged and damaged regions indicated by arrowheads $B$ and $C$ in Fig. $2 b$ for the SWCNT film after electrical discharge, respectively
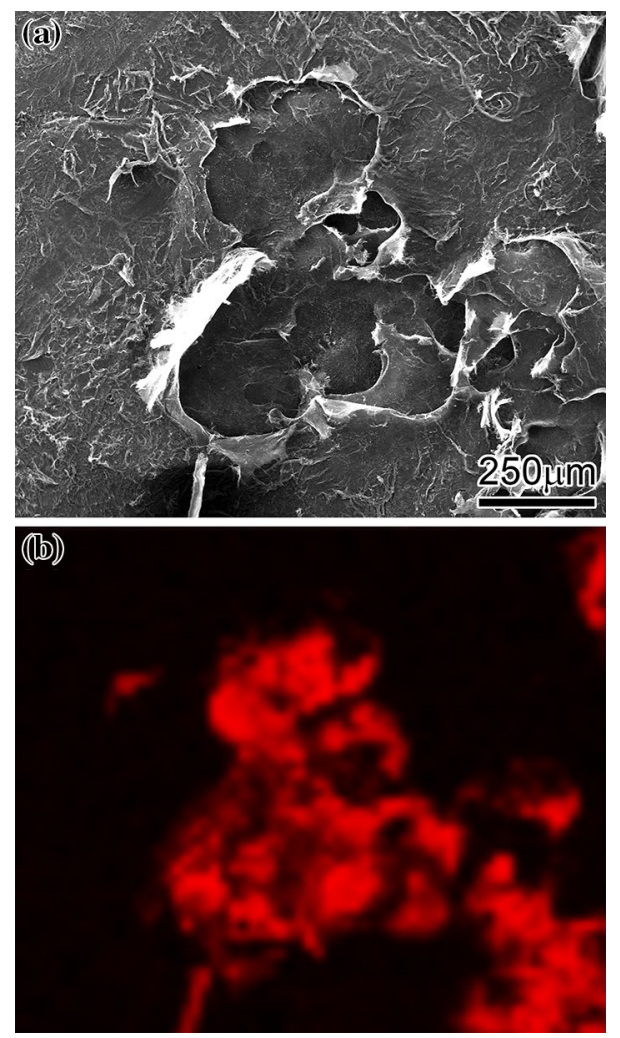

Fig. 4 a SEM image of the damaged region indicated by arrowhead $C$ in Fig. $2 b$, and $\mathbf{b}$ corresponding Raman mapping image using the L band in Fig. 3c. These peaks do not appear in the Raman spectra of as-prepared SWCNT films. On the other hand, linear carbon chains in carbon nanotubes are characterized by a Raman band in the $1780-1870 \mathrm{~cm}^{-1}$ range, which has been termed the $L$ band, and which is associated with C-C stretching-mode vibrations [37-40, 42-45, 48, 49]. The broad peak at around $600 \mathrm{~cm}^{-1}$ is also related to the linear carbon chains, because this peak is detected only when the $L$ band is observed $[40,48,49]$. There may be a possibility that the lower frequency peak at $600 \mathrm{~cm}^{-1}$ is attributed to bending modes of $\mathrm{sp}$ linear structures [50]. The simultaneous appearance of the marked peaks at 1863 and $600 \mathrm{~cm}^{-1}$ suggests the formation of linear atomic carbon chains in the SWCNT film upon electrical discharge. Figure $4 a$, b shows, respectively, an enlarged image of the damaged region indicated by arrowhead $\mathrm{C}$ in Fig. $2 \mathrm{~b}$ and the corresponding Raman mapping image using the $L$ band. The regions where the $L$ band signal is detected are colored red in Fig. $4 \mathrm{~b}$. The $L$ band is detected exclusively in the damaged regions of the SWCNT film. The major peak position of the $L$ band ranged from 1854 to $1865 \mathrm{~cm}^{-1}$, and its intensity and spectral shape differed, depending on the place in the damaged regions. The variation in the $L$ band may be attributed to the length and the encapsulating environment of the carbon chains [51]. The present results demonstrate that field electron emission accompanied by electrical discharge leads to the formation of linear carbon chains in the SWCNT film. It was also confirmed using another film that linear carbon chains are formed even after first electrical discharge during field emission, indicating that the repetition process of electrical discharge is not essential for the formation of the linear carbon chains. However, the damaged area suffered by the first electrical discharge is local in the SWCNT film and the yield of the carbon chains is low. In the present study, therefore, the repetition process of electrical discharge was carried out to form a large amount of carbon chains.

To demonstrate the presence of linear atomic carbon chains in the SWCNT films after electrical discharge, the structure of the SWCNT films was investigated by highresolution TEM. In addition to SWCNTs, the SWCNT films contained many DWCNTs that encapsulated linear atomic carbon chains. Figure 5 shows a typical high-resolution image of a DWCNT encapsulating a linear atomic carbon chain. The inner diameter of the DWCNT in Fig. 5 is $0.70 \mathrm{~nm}$, which is consistent with the reported innermost tube diameter of MWCNTs [37, 38, 41] and DWCNTs [45] encapsulating a carbon chain. As seen in Fig. 5 , the carbon chain is aligned along the central axis of the inner tube of the DWCNT, which is approximately at a van der Waals distance from the inner wall. The carbon chain as shown in Fig. 5 is longer than $21 \mathrm{~nm}$. Assuming that the lengths of single, double, and triple bonds in the chain 


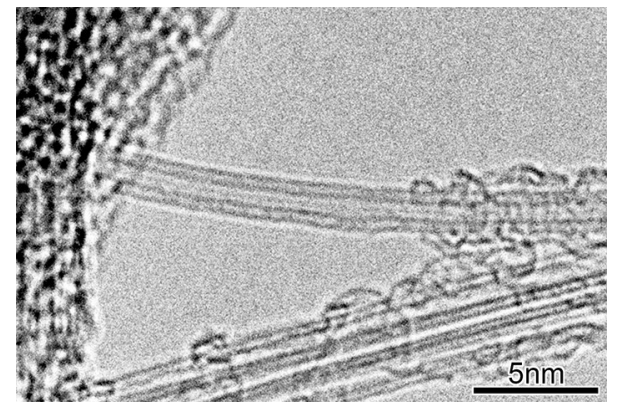

Fig. 5 High-resolution TEM image of a DWCNT with an inner diameter of $0.7 \mathrm{~nm}$ encapsulating a long linear atomic carbon chain along its central axis
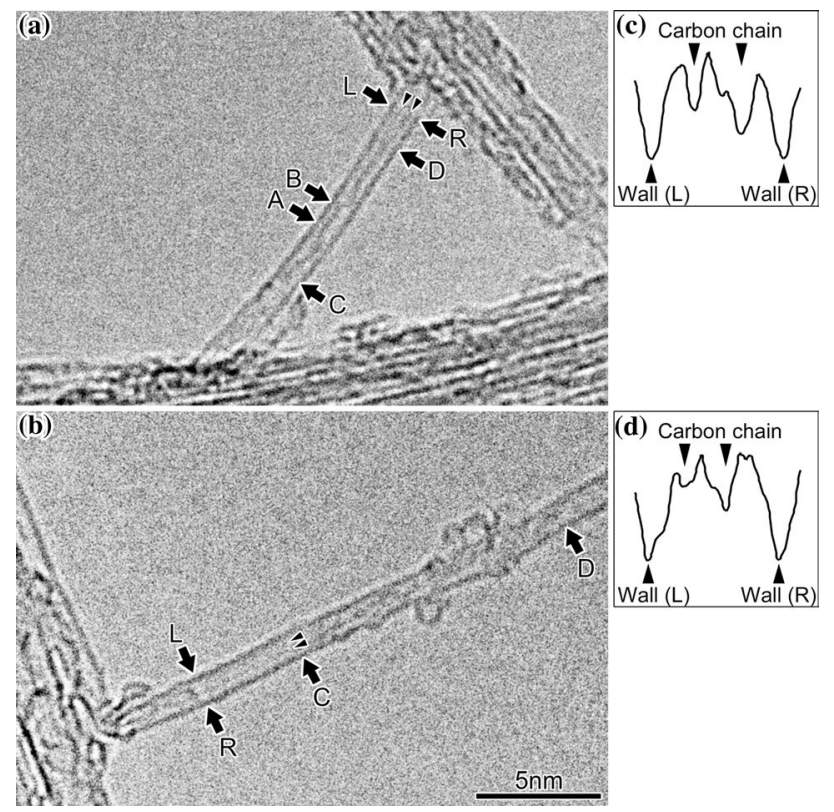

Fig. 6 a and b High-resolution TEM images of SWCNTs with a diameter of $1.0 \mathrm{~nm}$ encapsulating more than one carbon chain that were not completely linear and in an off-center position close to the inner wall. $\mathbf{c}$ and $\mathbf{d}$ Intensity profiles between the nanotube walls indicated by arrows $L$ and $R$ in (a) and (b), respectively

are, respectively, $0.1301,0.1282$, and $0.1265 \mathrm{~nm}$ [52] and the unit cell length of the carbon chain, which is twice the undistorted $\mathrm{C}-\mathrm{C}$ bond (double bond) length, is approximately $0.256 \mathrm{~nm}$, the carbon chain as shown in Fig. 5 is estimated to contain at least 160 contiguous carbon atoms.

Long linear carbon chains encapsulated in MWCNTs and DWCNTs have been reported previously [37-45]. In the present study, carbon chains encapsulated not only in DWCNTs but also in SWCNTs were observed, as previously reported [46]. Figure 6 shows two SWCNTs encapsulating carbon chains. The diameters of both SWCNTs are approximately $1.0 \mathrm{~nm}$, which is slightly larger than the inner diameter of the DWCNTs as shown in Fig. 5. Although the lengths of the carbon chains encapsulated in the SWCNTs in Fig. 6 are more than $14 \mathrm{~nm}$, their shape is not completely straight, as shown in Fig. 5 . The carbon chain in Fig. $6 a$ is bent at several locations, and portions of the chain, e.g., in the region between arrows $A$ and $B$, are positioned close to the nanotube wall, i.e., away from the central axis of the SWCNT. This deviation from the center probably results from the electronic $\pi-\pi$ interaction between the carbon chain and the SWCNT wall. The image contrast of the carbon chain in the region between arrows $C$ and $D$, as indicated in Fig. $6 a$, b, is as high as that of the nanotube wall, showing that the thickness of these carbon chains is not different from one-atom thickness of the carbon chain as shown in Fig. 5 . In the region on the right-hand side of arrow $D$ in Fig. 6a, the carbon chain branches off into two as indicated by arrowheads. The intensity profile between the nanotube walls indicated by arrows $L$ and $R$ in Fig. $6 a$ is shown in Fig. 6c, showing that the SWCNT encapsulates at least two carbon chains. In Fig. 6b, the two carbon chains are also seen within the SWCNT in the region on the left-hand side of arrow $C$, as shown by arrowheads. Thus, the high image contrast of the carbon chain in the region between arrows $C$ and $D$ in Fig. $6 a, b$ is thought to be due to the overlap of the carbon chains. The carbon chains may form cross-linking bonds between the chains, as previously reported [27, 34]. The formation of a single long linear carbon chain may require the sufficiently confined space of a carbon nanotube with a diameter of approximately $0.7 \mathrm{~nm}$.

In the present study, DWCNTs and SWCNTs that encapsulated carbon chains were observed in the morphologically damaged regions of the SWCNT films by electrical discharge during field emission. Although such destruction process by electrical discharge may play some kind of a role in the formation of the carbon chains inside the carbon nanotubes, it would be difficult to explain the formation mechanism of the carbon chains by only the destruction process. During field emission, the nanotubes experience high temperature [53]. As a possible mechanism, it may be conceivable that residual carbon left inside and/or outside a carbon nanotube aggregates and polymerizes in a narrow cavity of the nanotube to form a carbon chain by high-temperature heating and electrical discharge.

Although the formation mechanism for long linear carbon chains under high electric fields is still unclear, it is a fascinating topic for further study. The present results concerning the strong Raman spectral feature at $1863 \mathrm{~cm}^{-1}$ can potentially pave the way to a novel approach to the bulk production of long linear carbon chains involving a 
method other than the conventional high-temperature heat treatment of carbon nanotubes and arc discharge.

\section{Conclusion}

We carried out field electron emission experiments at a vacuum pressure of $8.3 \times 10^{-6} \mathrm{~Pa}$ using SWCNT films with a low degree of structural disorder for carbon networks and examined the structure of the SWCNT films after electrical discharge. The Raman spectroscopy and TEM results showed that field electron emission accompanied by electrical discharge from the SWCNT films induced the formation of long linear carbon chains, i.e., carbyne, within DWCNTs and SWCNTs. The present findings may lead to a new approach to synthesizing carbyne and promote carbyne research aimed at developing innovative applications.

Acknowledgements This study was partly supported by Research Foundation for the Electrotechnology of Chubu. The authors acknowledge Meijo Nano Carbon Co., Ltd. for supplying the SWCNT films.

\section{Compliance with ethical standards}

Conflict of interest On behalf of all authors, the corresponding author states that there is no conflict of interest.

\section{References}

1. Kertesz M, Koller J, Ažman A (1978) Ab initio Hartree-Fock crystal orbital studies. II. Energy bands of an infinite carbon chain. J Chem Phys 68:2779-2782

2. Casari CS, Tommasini M, Tykwinski RR, Milani A (2016) Carbonatom wires: 1-D systems with tunable properties. Nanoscale 8:4414-4435

3. Nair AK, Cranford SW, Buehler MJ (2011) The minimal nanowire: mechanical properties of carbine. Europhys Lett 95:16002

4. Liu M, Artyukhov VI, Lee H, Xu F, Yakobson BI (2013) Carbyne from first principles: chain of $C$ atoms, a nanorod or a nanorope. ACS Nano 7:10075-10082

5. Yang X, Huang Y, Cao B, To AC (2017) Length and temperature dependence of the mechanical properties of finite size carbine. Phys E 93:124-131

6. Zhang Y, Su Y, Wang L, Kong ES-W, Chen X, Zhang Y (2011) A onedimensional extremely covalent material: monatomic carbon linear chain. Nanoscale Res Lett 6:577

7. Wang M, Lin S (2015) Ballistic thermal transport in carbyne and cumulene with micron-scale spectral acoustic phonon mean free path. Sci Rep 5:18122

8. Liu X, Zhang G, Zhang Y-W (2015) Tunable mechanical and thermal properties of one-dimensional carbyne chain: phase transition and microscopic dynamics. J Phys Chem C 119:24156-24164

9. Lang ND, Avouris Ph (1998) Oscillatory conductance of carbonatom wires. Phys Rev Lett 81:3515-3518

10. Larade B, Taylor J, Mehrez H, Guo H (2001) Conductance, I-V curves, and negative differential resistance of carbon atomic wires. Phys Rev B 64:075420
11. Khoo KH, Neaton JB, Son YW, Cohen ML, Louie SG (2008) Negative differential resistance in carbon atomic wire-carbon nanotube junctions. Nano Lett 8:2900-2905

12. Dong $Y-J$, Wang $X-F$, Zhai M-X, Wu J-C, Zhou L, Han Q, Wu X-M (2013) Effects of geometry and symmetry on electron transport through graphene-carbon-chain junctions. J Phys Chem C 117:18845-18850

13. Akdim B, Pachter R (2011) Switching behavior of carbon chains bridging graphene nanoribbons: effects of uniaxial strain. ACS Nano 5:1769-1774

14. Artyukhov VI, Liu M, Yakobson BI (2014) Mechanically induced metal-insulator transition in carbine. Nano Lett 14:4224-4229

15. Zanolli Z, Onida G, Charlier J-C (2010) Quantum spin transport in carbon chains. ACS Nano 4:5174-5180

16. Fürst JA, Brandbyge $M$, Jauho A-P (2010) Atomic carbon chains as spin-transmitters: an ab initio transport study. Europhys Lett 91:37002

17. Zeng MG, Shen L, Cai YQ, Sha ZD, Feng YP (2010) Perfect spinfilter and spin-valve in carbon atomic chains. Appl Phys Lett 96:042104

18. Sorokin PB, Lee H, Antipina LYu, Singh AK, Yakobson BI (2011) Calcium-decorated carbyne networks as hydrogen storage media. Nano Lett 11:2660-2665

19. Lagow RJ, Kampa JJ, Wei HC, Battle SL, Genge JW, Laude DA, Harper CJ, Bau R, Stevens RC, Haw JF, Munson E (1995) Synthesis of linear acetylenic carbon: the "sp" carbon allotrope. Science 267:362-367

20. Chalifoux WA, Tykwinski RR (2010) Synthesis of polyynes to model the sp-carbon allotrope carbine. Nat Chem 2:967-971

21. Troiani HE, Miki-Yoshida M, Camacho-Bragado GA, Marques MAL, Rubio A, Ascencio JA, Jose-Yacaman M (2003) Direct observation of the mechanical properties of single-walled carbon nanotubes and their junctions at the atomic level. Nano Lett 3:751-755

22. Marques MAL, Troiani HE, Miki-Yoshida M, Jose-Yacaman M, Rubio A (2004) On the breaking of carbon nanotubes under tension. Nano Lett 4:811-815

23. Asaka K, Kizuka T (2005) Atomistic dynamics of deformation, fracture, and joining of individual single-walled carbon nanotubes. Phys Rev B 72:115431

24. Börrnert F, Börrnert C, Gorantla S, Liu X, Bachmatiuk A, Joswig J-O, Wagner FR, Schäffel F, Warner JH, Schönfelder R, Rellinghaus B, Gemming T, Thomas J, Knupfer M, Büchner B, Rümmeli MH (2010) Single-wall-carbon-nanotube/single-carbon-chain molecular junctions. Phys Rev B 81:085439

25. Asaka K, Karita M, Saito Y (2011) Joining of multiwall carbon nanotubes for the end-contact configuration by applying electric current. Mater Lett 65:1832-1834

26. Meyer JC, Girit CO, Crommie MF, Zettl A (2008) Imaging and dynamics of light atoms and molecules on graphene. Nature 454:319-322

27. Jin C, Lan H, Peng L, Suenaga K, lijima S (2009) Deriving carbon atomic chains from graphene. Phys Rev Lett 102:205501

28. Chuvilin A, Meyer JC, Algara-Siller G, Kaiser U (2009) From graphene constrictions to single carbon chains. New J Phys 11:083019

29. Song B, Schneider GF, Xu Q, Pandraud G, Dekker C, Zandbergen $\mathrm{H}$ (2011) Atomic-scale electron-beam sculpting of neardefect-free graphene nanostructures. Nano Lett 11:2247-2250

30. Cretu O, Botello-Mendez AR, Janowska I, Pham-Huu C, Charlier J-C, Banhart F (2013) Electrical transport measured in atomic carbon chains. Nano Lett 13:3487-3493

31. Robertson AW, Warner JH (2013) Atomic resolution imaging of graphene by transmission electron microscopy. Nanoscale 5:4079-4093 
32. La Torre A, Romdhane FB, Baaziz W, Janowska I, Pham-Huu C, Begin-Colin S, Pourroy G, Banhart F (2014) Formation and characterization of carbon-metal nano-contacts. Carbon 77:906-911

33. Kano E, Takeguchi M, Fujita J, Hashimoto A (2014) Direct observation of Pt-terminating carbyne on graphene. Carbon 80:382-386

34. Casillas G, Mayoral A, Liu M, Ponce A, Artyukhov VI, Yakobson $\mathrm{BI}$, Jose-Yacaman M (2014) New insights into the properties and interactions of carbon chains as revealed by HRTEM and DFT analysis. Carbon 66:436-441

35. La Torre A, Botello-Mendez A, Baaziz W, Charlier J-C, Banhart F (2015) Strain-induced metal-semiconductor transition observed in atomic carbon chains. Nat Commun 6:6636

36. Lin Y-C, Morishita S, Koshino M, Yeh C-H, Teng P-Y, Chiu P-W, Sawada $H$, Suenaga K (2017) Unexpected huge dimerization ratio in one-dimensional carbon atomic chains. Nano Lett 17:494-500

37. Zhao X, Ando Y, Liu Y, Jinno M, Suzuki T (2003) Carbon nanowire made of a long linear carbon chain inserted inside a multiwalled carbon nanotube. Phys Rev Lett 90:187401

38. Scuderi V, Scalese S, Bagiante S, Compagnini G, D'Urso L, Privitera V (2009) Direct observation of the formation of linear $C$ chain/carbon nanotube hybrid systems. Carbon 47:2112-2142

39. Scalese S, Scuderi V, Bagiante S, Simone F, Russo P, D'Urso L, Compagnini G, Privitera V (2010) Controlled synthesis of carbon nanotubes and linear $C$ chains by arc discharge in liquid nitrogen. J Appl Phys 107:014304

40. Andrade NF, Vasconcelos TL, Gouvea CP, Archanjo BS, Achete CA, Kim YA, Endo M, Fantini C, Dresselhaus MS, Filho AGS (2015) Linear carbon chains encapsulated in multiwall carbon nanotubes: Resonance Raman spectroscopy and transmission electron microscopy studies. Carbon 90:172-180

41. Wang Z, Ke X, Zhu Z, Zhang F, Ruan M, Yang J (2000) Carbonatom chain formation in the core of nanotubes. Phys Rev $B$ 61:R2472

42. Cazzanelli E, Castriota M, Caputi LS, Cupolillo A, Giallombardo C, Papagno $L$ (2007) High-temperature evolution of linear carbon chains inside multiwalled nanotubes. Phys Rev B 75:121405R

43. Zhao C, Kitaura R, Hara H, Irle S, Shinohara H (2011) Growth of linear carbon chains inside thin double-wall carbon nanotubes. J Phys Chem C 115:13166-13170
44. Zhang J, Feng Y, Ishiwata H, Miyata Y, Kitaura R, Dahl JEP, Carlson RMK, Shinohara H, Tománek D (2012) Synthesis and transformation of linear adamantane assemblies inside carbon nanotubes. ACS Nano 6:8674-8683

45. Shi L, Rohringer $P$, Suenaga $K$, Niimi $Y$, Kotakoski J, Meyer JC, Peterlik H, Wanko M, Cahangirov S, Rubio A, Lapin ZJ, Novotny L, Ayala P, Pichler T (2016) Confined linear carbon chains as a route to bulk carbine. Nat Mater 15:634

46. Toma S, Asaka K, Irita M, Saito Y (2017) Bulk synthesis of linear carbon chains confined inside single-wall carbon nanotubes by vacuum discharge. Surf Interface Anal 54:131-135

47. Spindt CA, Brodie I, Humphrey L, Westerberg ER (1976) Physical properties of thin-film field emission cathodes with molybdenum cones. J Appl Phys 47:5248-5263

48. Jinno M, Bandow S, Ando Y (2004) Multiwalled carbon nanotubes produced by direct current arc discharge in hydrogen gas. Chem Phys Lett 398:256-259

49. Jinno M, Ando Y, Bandow S, Fan J, Yudasaka M, lijima S (2006) Raman scattering study for heat-treated carbon nanotubes: the origin of $\approx 1855 \mathrm{~cm}^{-1}$ Raman band. Chem Phys Lett 418:109-114

50. Casari CS, Bassi AL, Baserga A, Ravagnan L, Piseri $P$, Lenardi $C$ Tommasini M, Milani A, Fazzi D, Bottani CE, Milani P (2008) Lowfrequency modes in the Raman spectrum of $s p-s p^{2}$ nanostructured carbon. Phys Rev B 77:195444

51. Wanko M, Cahangirov S, Shi L, Rohringer P, Lapin ZJ, Novotny L, Ayala P, Pichler T, Rubio A (2016) Polyyne electronic and vibrational properties under environmental interactions. Phys Rev B 94:195422

52. Cahangirov S, Topsakal M, Ciraci S (2010) Long-range interactions in carbon atomic chains. Phys Rev B 82:195444

53. Wei W, Liu Y, Wei Y, Jiang K, Peng LM, Fan S (2007) Tip cooling effect and failure mechanism of field-emitting carbon nanotubes. Nano Lett 7:64-68

Publisher's Note Springer Nature remains neutral with regard to jurisdictional claims in published maps and institutional affiliations. 